\title{
Australasian Pediatric Gastroenterologists' Perspectives and Practices of Celiac Disease Diagnosis and Management
}

\author{
Shaun S. C. Ho ${ }^{1}$ Sophie Hall ${ }^{1}$ Jacqueline I. Keenan ${ }^{2} \cdot$ Andrew S. Day $^{1,3}$ (]) \\ Received: 12 October 2020 / Accepted: 30 March 2021 / Published online: 30 April 2021 \\ (c) The Author(s), under exclusive licence to Springer Science+Business Media, LLC, part of Springer Nature 2021
}

\begin{abstract}
Background The application of the European Society for Paediatric Gastroenterology Hepatology and Nutrition (ESPGHAN) celiac disease $(\mathrm{CeD})$ guidelines by pediatric gastroenterologists in Australia and New Zealand (Australasia) is unknown. Similarly, long-term management practices for patients with $\mathrm{CeD}$ are also unknown in this region.

Aims This study aimed to explore the perceptions and practices of Australasian pediatric gastroenterologists in diagnosing and managing patients with $\mathrm{CeD}$.

Methods Australasian pediatric gastroenterologists and trainees were invited to complete an anonymous online survey over a 3-week period.

Results The survey was completed by 28 respondents, 24 from Australia and four from New Zealand. Tissue transglutaminase antibody IgA was the most frequently ordered initial serologic test. Fifteen (54\%) respondents relied on duodenal biopsies for the confirmation of $\mathrm{CeD}$, six (21\%) followed the ESPGHAN guidelines and the remaining seven offered either biopsy confirmation or no-biopsy diagnosis according to the parents' wishes. Following diagnosis, five (18\%) respondents discharged patients from care, three (11\%) discharged patients after one follow-up visit, one (4\%) reviewed patients for 12 months, six (21\%) reviewed patients until celiac antibodies normalized and children were clinically asymptomatic, and 13 (46\%) reviewed patients until transition to adult care.

Conclusion Tissue transglutaminase antibody IgA was the most common initial serologic test ordered by this group of Australasian pediatric gastroenterologists. Half of these physicians rely solely on duodenal biopsy for the confirmation of CeD diagnosis: a minority routinely use the ESPGHAN guidelines. Physicians reported a wide range of CeD follow-up practices.
\end{abstract}

Keywords Celiac disease $\cdot$ Pediatrics $\cdot$ Diagnosis $\cdot$ Gastroenterologist $\cdot$ Follow-up $\cdot$ ESPGHAN

Andrew S. Day

andrew.day@otago.ac.nz

Shaun S. C. Ho

shaunsc.ho@gmail.com

Sophie Hall

Sophiewhall@gmail.com

Jacqueline I. Keenan

jacqui.keenan@otago.ac.nz

1 Department of Paediatrics, University of Otago, 2 Riccarton Avenue, Christchurch 8011, New Zealand

2 Department of Surgery, University of Otago, 2 Riccarton Avenue, Christchurch 8011, New Zealand

3 Department of Paediatrics, University of Otago, Riccarton Avenue, Christchurch 8140, New Zealand

\section{Introduction}

Celiac disease (CeD), an immune-mediated enteropathy triggered by recurrent exposure to gluten in genetically susceptible individuals, is recognized worldwide [1,2]. The prevalence of $\mathrm{CeD}$ in Australasian (Australia and New Zealand (NZ) adults is approximately $1.2 \%$ [3, 4]. A rising rate, however, has been noted in NZ children [5].

$\mathrm{CeD}$ presentations in children can range from typical gastrointestinal malabsorptive symptoms to asymptomatic, and children suspected of having $\mathrm{CeD}$ are required to undertake at least an initial celiac serologic test. Conventionally, any children with positive celiac antibodies also require an intestinal biopsy for CeD confirmation. In 2012, the European Society of Paediatric Gastroenterology, Hepatology and Nutrition (ESPGHAN) revised their CeD diagnostic guidelines (ESPGHAN guidelines) to incorporate a 
no-biopsy pathway for a subgroup of children who fulfilled their criteria [6]. More recently (available from late 2019 as early online access), the ESPGHAN guidelines were further updated, expanding the no-biopsy criteria (tissue transglutaminase antibody IgA (TGA IgA) $\geq 10$-fold the upper limit of normal and positive endomysial antibodies IgA (EMA IgA)) to include all children regardless of symptoms at presentation or at-risk groups, while the requirement of celiac HLA typing as a part of the no-biopsy pathway was also omitted [7]. The utilization of the no-biopsy pathway being part of the ESPGHAN guidelines has increasingly been recommended in worldwide clinical practices, including during the COVID-19 pandemic [8-10]. However, the influence of such guidelines among the practices of Australasian pediatric gastroenterologists is unknown.

The only current treatment for individuals diagnosed with $\mathrm{CeD}$ is a strict life-long gluten-free diet (GFD). Currently, there is limited literature on the best-practice guidance for the long-term management of patients with $\mathrm{CeD}$. A number of international organizations and experts have provided their recommendations with some similarities and variations as summarized by Hall and Day [11]. There is no previous published literature on the follow-up practices of children diagnosed with $\mathrm{CeD}$ by Australasian pediatric gastroenterologists.

In view of the increased acceptance of ESPGHAN guidelines in practice and the interest of long-term management of children with $\mathrm{CeD}$, a cross-sectional survey was conducted involving Australian and NZ pediatric gastroenterologists. The study aimed to explore the practices and perspectives of the gastroenterologists with regard to screening for and diagnosing $\mathrm{CeD}$ and also how they subsequently manage their patients with $\mathrm{CeD}$.

\section{Methods}

\section{Participants}

Australian and NZ pediatric gastroenterologists including trainees were invited via an email listserv of the Australasian Society of Paediatric Gastroenterology, Hepatology and Nutrition (AuSPGHAN) bulletin board. This email listserv bulletin board is a closed group and consisted of 78 currently practicing or previous pediatric gastroenterologists and trainees who worked/trained in Australia or New Zealand. It was not possible to determine the members' practicing geographical location through the email listserv.

\section{Anonymous Online Survey}

The anonymous online survey was conducted using an online platform, Qualtrics ${ }^{\circledR}$ Version 2019 (Utah, USA) and ran over a 3-week period (15 November 2019 till 5 December 2019). Reminder emails were sent at weekly intervals via the AuSPGHAN bulletin board. Participants accessed the survey using Qualtrics ${ }^{\odot}$ weblink provided in the invitation email. The weblink opened an external web browser providing participants with survey participation information before they proceed to undertake the survey. The questionnaire consisted of three themes: celiac screening practices, diagnostic methods (intestinal biopsy and/or ESPGHAN guidelines) used to confirm $\mathrm{CeD}$ and follow-up practices for those children confirmed to have $\mathrm{CeD}$ (Supplementary Questionnaire). Reasons for and against why respondents chose to or not to routinely use the ESPGHAN guidelines were sought. At the end of the survey, respondents had the option of providing their demographic details.

This study was approved by the subcommittee of the University of Otago Human Ethics Committee (Health).

\section{Statistical Analysis}

Data were exported from Qualtrics ${ }^{\odot}$ into IBM SPSS Statistics version 26.0 (IBM Corp., Armonk, NY, USA) for descriptive statistical analysis. Results were expressed as median \pm interquartile range (IQR). Fisher's exact test was used to analyze contingency tables. A $p$ value of less than 0.05 was considered statistically significant.

\section{Results}

\section{Background of Respondents}

Twenty-eight practicing Australasian pediatric gastroenterologists and trainees completed the survey. Of the 28 respondents, $24(82 \%)$ were from Australia and 4 (18\%) were from New Zealand. All respondents provided their background details (Table 1).

\section{Celiac Screening Practices}

Twenty-six respondents (93\%) chose TGA IgA as their most frequently ordered initial celiac serology test for children of any age (Fig. 1). Deamidated anti-gliadin peptide IgG (DGP IgG) was ordered significantly more frequently in children under two years of age than in children over two years old, ( 20 of $28,71 \%$ versus 13 of $28,46 \%(p=0.04)$, respectively). Up to a third of the physicians reported that other celiac serologic tests including tissue transglutaminase antibody $\operatorname{IgG}$ (TGA IgG), EMA IgA and deamidated anti-gliadin peptide $\operatorname{Ig}$ A (DGP IgA) were also ordered for children suspected of having CeD.

There was no difference in the frequency of the initial celiac serology tests ordered between the respondents of the 
Table 1 Background characteristics of the 28 respondents who completed the survey

\begin{tabular}{ll}
\hline & $N(\%)$ \\
\hline Male & $24(86)$ \\
Age (years) & \\
$<30$ & 0 \\
$30-40$ & $6(21)$ \\
$41-50$ & $11(39)$ \\
$51-60$ & $6(21)$ \\
$>60$ & $5(18)$ \\
Australia & $24(86)$ \\
New South Wales & 7 \\
Queensland & 2 \\
South Australia & 5 \\
Victoria & 9 \\
Western Australia & 1 \\
New Zealand & $4(14)$ \\
Auckland & 4 \\
Practice & \\
Public hospital/academic & $24(86)$ \\
Private & $18(64)$ \\
Position & $26(93)$ \\
Consultant & $2(7)$ \\
Advanced trainee (fellow) &
\end{tabular}

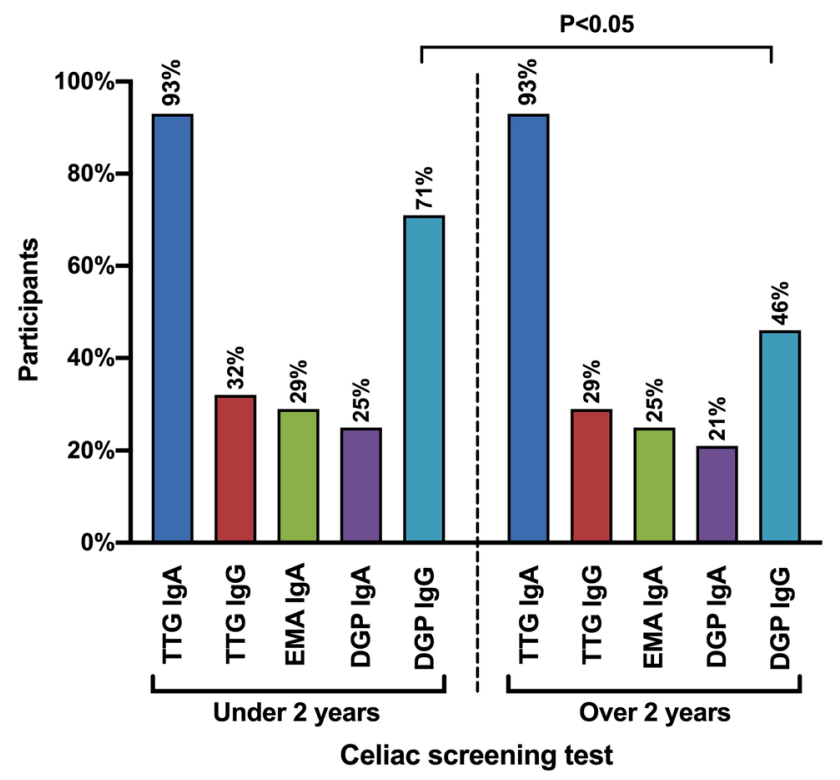

Fig. 1 Views of 28 Australian and New Zealand pediatric gastroenterologists on their practices of ordering celiac screening tests including anti-tissue transglutaminase IgA (TTG IgA), anti-tissue transglutaminase IgG (TTG IgG), endomysial antibodies IgA (EMA IgA), deamidated anti-gliadin peptide $\operatorname{IgA}$ (DGP IgA) and deamidated antigliadin peptide IgG (DGP IgG) in children suspected of having celiac disease. DGP IgG test was preferred in children under 2 years of age (Fisher's exact, $p<0.05$ ) two countries (data not shown). Moreover, all but two of the respondents reported routinely requesting total IgA levels. Of these two, one respondent reported this was because the laboratory routinely performs the test. The other respondent did not provide a reason.

In addition to celiac antibody tests, gastroenterologists also frequently ordered iron studies (96\%) and a full blood count (93\%) as part of the screening investigations (Supplementary Figure 1). Only one respondent reported not routinely ordering any additional tests.

\section{Celiac Diagnostic Practices}

Among all respondents, 15 (54\%) relied on duodenal biopsies for confirmation of $\mathrm{CeD}$ diagnosis and six $(21 \%)$ followed the ESPGHAN guidelines (three followed 2012 ESPGHAN guidelines [6] and three followed 2020 ESPGHAN guidelines [7]). The remaining seven respondents offered both options (either intestinal biopsy confirmation or no-biopsy 2012 ESPGHAN guidelines (if criteria fulfilled)), according to the parents' wishes (Fig. 2a).

The celiac diagnostic practices were different between the two countries. Almost two-thirds of the Australian respondents $(N=15,63 \%)$ relied solely on intestinal biopsies (Fig. 2b). In contrast, three-quarters of the NZ respondents $(N=3)$ used the ESPGHAN guidelines only and one respondent offered both options (biopsy confirmation or no-biopsy ESPGHAN guidelines). A quarter $(N=6)$ of the Australian gastroenterologists offered both options and the remaining 22\% followed the ESPGHAN guidelines only.

When intestinal biopsies were required, all endoscopists reported that at least one mucosal biopsy was obtained from the first part of duodenum and at least two biopsies from either second or third parts of the duodenum. In addition, one respondent took biopsies from the fourth part of duodenum and two others took biopsies from the jejunum (Supplementary Table 1).

\section{Respondents' Perspectives on the Application of ESPGHAN Guidelines in Clinical Practice}

Of the six respondents who claimed to use an ESPGHAN guideline in their practice, all felt that there was good evidence to support practice and that this helped to reduce the need for endoscopy (Fig. 3). Four gastroenterologists also felt the guidelines help to reduce endoscopy waiting time. Additional reported benefits included support from peers $(N=2)$, hospital $(N=2)$ and laboratory services $(N=3)$.

Of the 15 respondents who claimed not to use the ESPGHAN guidelines in their practice, the most frequent reason for not using the guidelines was due to physicians' personal experience with false positive celiac serology results $(N=10)$. Six clinicians felt there was insufficient 
Fig. 2 Celiac diagnostic practices. a Methods used by all respondents to confirm celiac disease in children. b Comparing diagnostic practices between Australia and New Zealand respondents
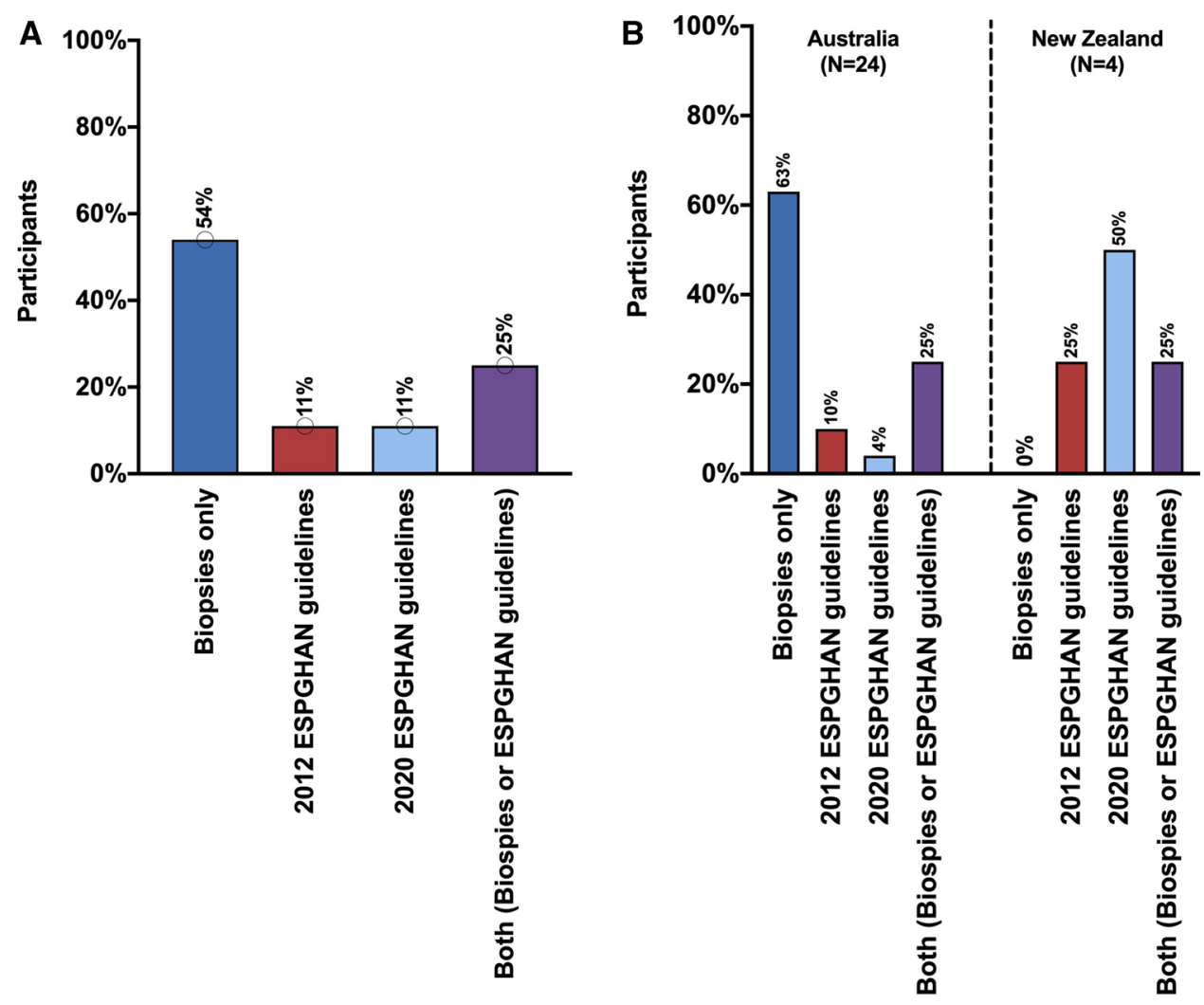

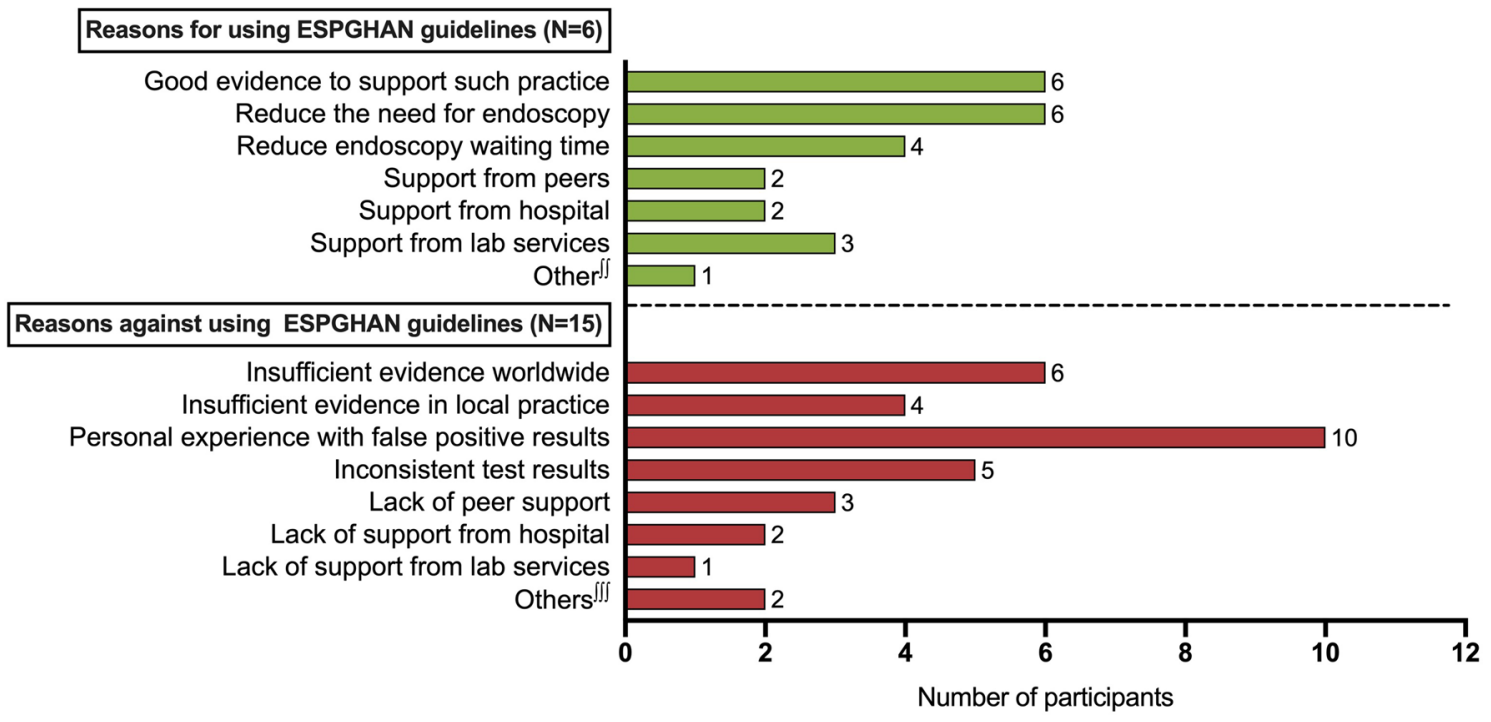

Fig. 3 Respondents' perspectives of applying the ESPGHAN celiac disease diagnostic guidelines in their practice. $\iint$ Other, prospective trial to validate local population $(N=1)$; $\iiint$ Others, life-long disease, confirm with biopsies $(N=1)$ and hospital practice $(N=1)$

evidence worldwide, and four others felt that there was inadequate local evidence. Inconsistent test results were mentioned by five respondents. Other reasons provided included lack of support from peers $(N=3)$, hospital $(N=2)$, or laboratory services $(N=1)$.

\section{Follow-Up Following Diagnosis of Celiac Disease}

Following receipt of the celiac diagnostic investigation results, $71 \%$ of respondents would offer face-to-face consultation and $54 \%$ would call parents or patients to discuss 
Fig. 4 Follow-up practices reported by 28 respondents following celiac diagnostic investigations. a Initial follow-up practices including referrals and communications. b Long-term follow-up practices following initial follow-up
A

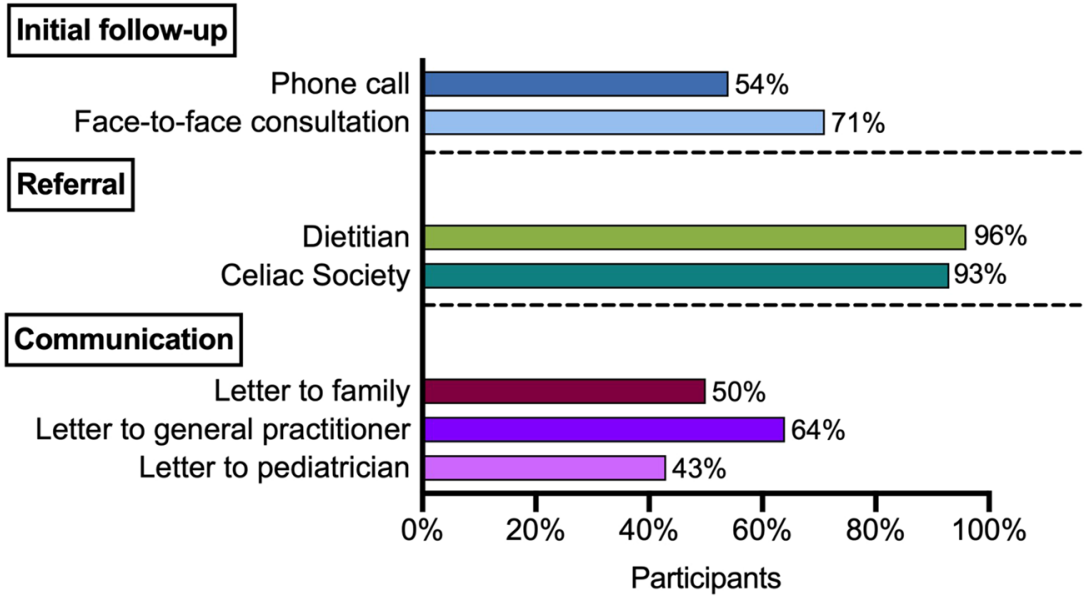

B

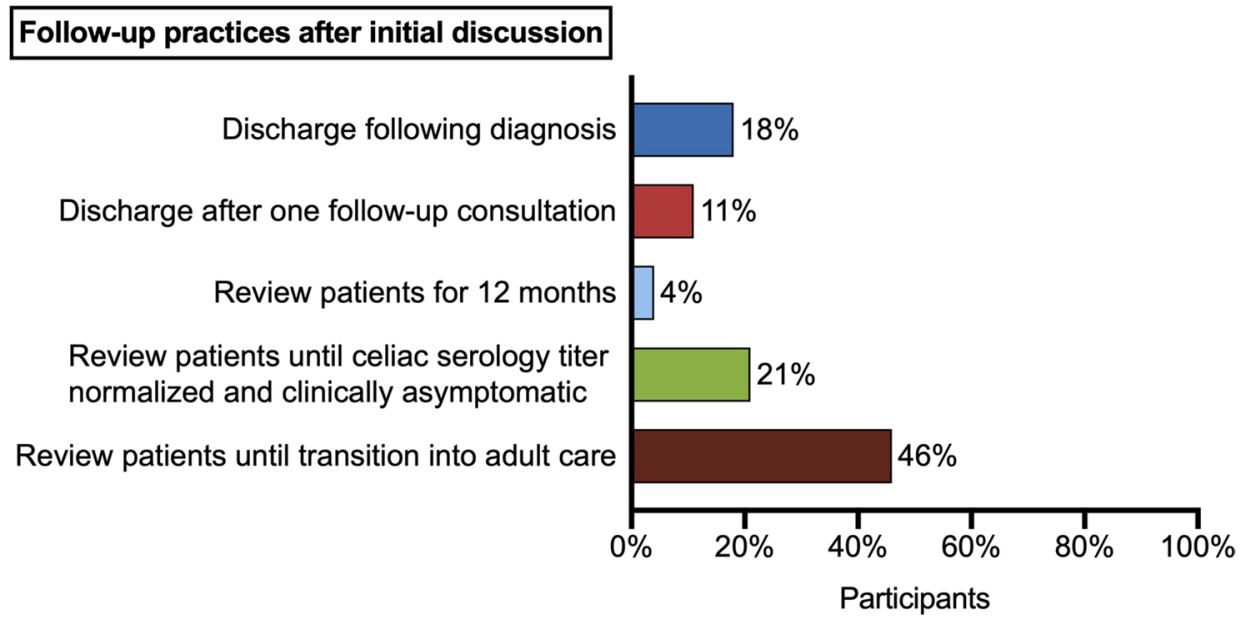

their results, with some offering both options (Fig. 4a). Furthermore, $96 \%$ of physicians would refer their patients to see a dietitian and 93\% to their local Celiac Society. A written letter was also completed by $50 \%$ of respondents to the family, $64 \%$ to the patient's general practitioner, and $43 \%$ to the patient's pediatrician (if any).

Almost half of the respondents (46\%) continued to review their patients diagnosed with $\mathrm{CeD}$ until transition into adult care (Fig. 4b). A smaller number (21\%) of clinicians follow patients until their celiac serology titer has normalized and the children were clinically well. Eighteen percent of gastroenterologists discharged patients from their care following diagnosis, $11 \%$ offered one follow-up consultation before discharging from care, while $4 \%$ reviewed their patients for 12 months.

The twenty respondents who continued to review their patients regularly after $\mathrm{CeD}$ diagnosis were asked about the assessments and discussions that occurred during follow-up visits. The majority of respondents (95\%) assessed their patient's adherence to a GFD and growth
(Supplementary Figure 2). Others screened for micronutrient deficiencies $(80 \%)$ and autoimmune comorbidities $(75 \%)$, discussed potential future treatment $(75 \%)$, discussed healthy diet (50\%), and lastly, some included a discussion about maximizing the patient's quality of life (40\%). Unfortunately, one respondent did not provide any response.

Moreover, 18 of 20 respondents within this group would request blood tests prior to a follow-up visit (Supplementary Figure 3). TGA IgA (89\%) was among the most commonly requested celiac serology test during routine follow-up. All respondents screened for iron deficiency. Thyroid function testing ( $78 \%$ of respondents) was among the most ordered test for screening autoimmune comorbidities.

When patients were discharged from gastroenterology care, all respondents referred patients back to their general practitioner, except for those who were reviewed until transition into adult care. Of the 13 pediatric gastroenterologists who continued to review patients until adulthood, $62 \%$ referred patients back to the general practitioner, $23 \%$ to an 
adult gastroenterologist, and $15 \%$ to both general practitioner and an adult gastroenterologist.

\section{Discussion}

The current study identified wide variations in the practices of Australasian pediatric gastroenterologists with regard to screening children for $\mathrm{CeD}$, diagnosis of $\mathrm{CeD}$, and subsequent review of children with CeD. TGA IgA was the most frequently ordered initial serology test in any child suspected of CeD. Overall, half the respondents relied solely on duodenal biopsies for the diagnosis of $\mathrm{CeD}$. However, NZ respondents reported they only relied on either the ESPGHAN guidelines or offered both options (intestinal biopsy confirmation or no-biopsy ESPGHAN CeD diagnosis) according to the parents' wishes. Follow-up practices were also widely reported, with almost half the physicians reviewing their patients until adulthood, whereas others discharged patients from their care at various times after diagnosis. For those who routinely reviewed children with $\mathrm{CeD}$, the management involving assessments and discussions varied during visit.

TGA IgA was the most frequently ordered initial serology test by this group of pediatric gastroenterologists to screen $\mathrm{CeD}$ in children of all ages. This finding is aligned with international guidelines recommending TGA IgA as the first line of testing $[7,8,12-18]$, with the majority also recommending simultaneous measurement of total $\operatorname{IgA}$ levels [7, 8, 12, 14, 16-18]. In contrast, the World Gastroenterology Organisation (WGO) guideline recommends that either TGA IgA or EMA IgA or both can be used for initial testing (without specifying an age grouping) [13].

In addition to ordering TGA $\operatorname{IgA}$, this study found that the DGP IgG test was more frequently ordered for children under two years of age compared to those over two years of age. IgA-based serology tests are noted to be less sensitive in young children consequent to immature IgA responses $[19,20]$. The most recent revised 2020 ESPGHAN guideline recommends testing for total IgA and TGA IgA in children of any age and suggests that IgG-based serology tests (DGP, EMA or TGA) are considered only in those with low IgA levels [7]. The authors of these guidelines felt that the combination of various IgA- and IgG-based serology tests did not improve test sensitivity once patients with low IgA levels were excluded [7]. The recommendation of using TGA IgA as the initial serology test in all children is further supported by a recent North American multicenter retrospective study [21].

Despite the inclusion of the no-biopsy pathway in the ESPGHAN guidelines, half of the Australasian gastroenterologists included in this study reported that, they still rely solely on biopsy as a confirmatory test for CeD diagnosis. Although six (21\%) respondents reported they routinely follow the ESPGHAN guidelines, half of them followed the latest ESGPHAN CeD guidelines. In the latest 2020 ESGPHAN CeD guidelines, it is mentioned that the physician should discuss the two options (biopsy or no-biopsy) of diagnosing $\mathrm{CeD}$ with the patient/parents if the patient fulfils the no-biopsy criteria [7]. For this reason, those respondents who chose to follow the 2020 ESPGHAN guidelines can be thought to fall in the same category as those respondents who offered both biopsy and no-biopsy options (when 2012 ESPGHAN criteria fulfilled) according to parental wishes. These results were intentionally not merged together to avoid confusion.

When the practices of diagnosing $\mathrm{CeD}$ were stratified by country, none of the NZ respondents relied solely on biopsy for $\mathrm{CeD}$ confirmation compared to almost two-thirds of the Australian physicians who continued to rely solely on biopsy for disease confirmation. It is important to note that there were a low number of participants from NZ and all were located in Auckland, where this region had prospectively studied the efficacy of utilizing the 2012 ESPGHAN guidelines in their local population [22]. It is interesting to note, however, that since the current survey was conducted, a Western Australian study has been published that reports prospectively applying the 2020 ESPGHAN guidelines to their local population [23], suggesting that local practice may be changing in this state.

Although not all respondents provided their views about the ESPGHAN guidelines, all six respondents who used the ESPGHAN guidelines in their practice believed there is sufficient evidence to apply such practice locally. Meanwhile, two-thirds of the clinicians who did not use the guidelines had experience of false positive celiac serology results, six responders felt there was insufficient evidence worldwide, and four others felt inadequate evident in their local practice. Only one respondent reported that a prospective study was carried out locally to support the implementation of the ESPGHAN guidelines. The present survey did not investigate the specific aspects of insufficient evidence and should be explored in future studies, including whether the false positive results were due to antibody titers less or $\geq 10$-fold the upper limit of normal. However, variable standardization in laboratory assay and references may play a role in the implementation of such guidelines [24]. Hence, it is crucial that findings from one region cannot be generalized to other regions of the same country or to another country. On the other hand, the current survey also found regular users of the ESPGHAN guidelines believed the integration of the no-biopsy pathway helps to reduce the need for endoscopy. This is supported by a number of studies demonstrating the potential extent of endoscopy reduction up to $60 \%[9,22$, 23, 25-27].

Predictably, the present group of physicians reported an extensive range of follow-up practices. This variability is 
likely consequent to the paucity of evidence-based followup protocols for children with CeD. While the current study only explored the follow-up practices of the individual respondents, the rationales supporting such practices should be considered in future studies. International guidelines and experts do recommend children with $\mathrm{CeD}$ should be followed up following their diagnosis $[8,12-18,28]$. One study reported that children with $\mathrm{CeD}$ who are lost to follow-up have reduced adherence to GFD (regression analysis $=0.27$, $p=0.001)$ and a higher prevalence of positive celiac serology tests $(50 \%$ compared to $25 \%$ of regular follow-up patients, $p=0.01$ ) [29]. This highlights the importance of follow-up following $\mathrm{CeD}$ diagnosis. However, as to which healthcare professional (dietitian, general practitioner, adult gastroenterologist or general physician with CeD interest) is best suited to continue care once adulthood is achieved remains unclear. In a 28 -year follow-up study of 50 adults who were diagnosed with $\mathrm{CeD}$ during childhood, the authors found that a third were not adherent to their GFD and only $22 \%$ were enrolled in an adult gastroenterology clinic [30]. These patients did not receive any medical or dietary supervision after transition to adulthood. Recently, two adult transfer of care models were introduced, but neither has been prospectively validated [31, 32].

In a different approach, Sbravati et al. [33] prospectively assessed the GFD adherence of 200 children over two intervals (at least 24 months) following transition from a single referral center to a general pediatrician once remission was achieved. Adherence was assessed using the TGA IgA and Biagi Score [34]. The study found such practice is a reasonable approach to ongoing management of $\mathrm{CeD}$ [33]. However, in this study, age over 13 years and nonItalian ethnicity were both found to be associated with GFD non-adherence. Hence, the authors advocated that specific attention is required for adolescents and foreign nationalities when counseling on GFD is required.

For those clinicians who regularly reviewed children with $\mathrm{CeD}$, the top five assessments and discussions conducted were: assessing patient's adherence to a GFD, growth, screening for micronutrient deficiencies and autoimmune comorbidities, and discussion about potential future treatments. All of the organization guidelines and expert reports emphasize the importance of assessing adherence to a GFD during follow-up [8, 12-18]. However, not all guidelines provide recommendations on assessing growth, screening for micronutrient deficiencies, or autoimmune comorbidities [11]. Specifics on how GFD adherence was assessed by clinicians were not explored in this survey. Resolution of symptoms was not specifically asked in the present study as the study focused on routine assessments and discussions occurring in a follow-up visit when the patient is asymptomatic.

Most international guidelines do not recommend repeat duodenal biopsies in children with $\mathrm{CeD}$ during follow-up unless the celiac serology remains persistently high or minimal changes and/or non-resolution of symptoms [12, 14-17]. Instead, celiac serology is used in conjunction with adherence assessment by the clinician with/without dietitian input. All guidelines suggest at least testing TGA antibodies during follow-up with some variations among their recommendations $[8,12-18]$. The current study findings of TGA IgA being the most frequently ordered initial test during follow-up are aligned with the guidelines.

This is the first report on the perspectives and practices of CeD by Australasian pediatric gastroenterologists. Nevertheless, the study has some limitations. Firstly, the survey was conducted prior to the COVID-19 pandemic, during which many healthcare systems have changed to adapt to the new challenges. The practices of the physicians in the region may have changed in the face of these events. Secondly, this survey was performed about a month after the 2020 ESPGHAN $\mathrm{CeD}$ diagnosis guideline preprint was released, but prior to the in-print publication date. This survey was not designed to assess the respondent's knowledge or awareness of the two guidelines, nor was it designed to assess when practitioners may have changed their practice. Thirdly, the design of the study did not enable clarification of several aspects and did not capture the attitudes of other disciplines, such as dietitian involvement in the follow-up practices. More comprehensive understanding could be obtained if data such as patient volume and serology tests ordered could be matched with a physician's response, compared to a self-reported survey. A limited array of options for answers in the survey may have been misinterpreted by participants, especially trainee or clinicians with low patient load. In addition, the use of an online survey following an email invitation may have led to a selection bias. The total number of pediatric gastroenterologists and trainees in Australia is substantially larger than in $\mathrm{NZ}$, meaning that the findings of the study may be biased more toward Australian practices. However, the geographical spread of the respondents suggests that the findings are likely representative of the relevant regions, especially as all the NZ respondents were from the Auckland region. The nature of the bulletin board system means that was not possible to ascertain the current employment status of the respondents or to confirm their geographical location.

In conclusion, this study highlights key similarities and some variations in $\mathrm{CeD}$ practice by this group of pediatric gastroenterologists across Australasia. Overall, the ESPGHAN guidelines were not widely used in Australasia. In addition, physicians reported a wide range of $\mathrm{CeD}$ follow-up practices. Further studies are needed to compare the effectiveness of different diagnostic and management strategies to establish best practice guidance for children with $\mathrm{CeD}$. 
Supplementary Information The online version contains supplementary material available at https://doi.org/10.1007/s10620-021-06988-2.

Acknowledgments We would like to acknowledge Freemasons New Zealand for their support of SSCH via the Freemasons Paediatric Postgraduate Scholarship.

\section{Declarations}

Conflict of interest SSCH received his scholarship from Freemasons New Zealand. No further disclosures.

Research involving human participation and/or animals This study was approved by the subcommittee of the University of Otago Human Ethics Committee (Health). This study did not involve any direct patient contact.

Informed consent Informed consent was not obtained from participants in this study as the survey was conducted anonymously. Participants were given the option of providing their demographic details at the end of the survey.

\section{References}

1. Fasano A, Catassi C. Clinical practice. Celiac disease. $N$ Engl J Med 2012;367:2419-2426. https://doi.org/10.1056/NEJMcp1113 994.

2. Singh P, Arora A, Strand TA, Leffler DA, Catassi C, Green PH et al. Global prevalence of celiac disease: systematic review and meta-analysis. Clin Gastroenterol Hepatol 2018;16:823-36 e2. https://doi.org/10.1016/j.cgh.2017.06.037.

3. Anderson RP, Henry MJ, Taylor R, Duncan EL, Danoy P, Costa $\mathrm{MJ}$ et al. A novel serogenetic approach determines the community prevalence of celiac disease and informs improved diagnostic pathways. BMC Med 2013;11:188. https://doi.org/10.1186/ 1741-7015-11-188.

4. Cook HB, Burt MJ, Collett JA, Whitehead MR, Frampton CM, Chapman BA. Adult coeliac disease: prevalence and clinical significance. J Gastroenterol Hepatol 2000;15:1032-1036. https:// doi.org/10.1046/j.1440-1746.2000.02290.x.

5. Kho A, Whitehead M, Day AS. Coeliac disease in children in Christchurch, New Zealand: presentation and patterns from 20002010. World J Clin Pediatr 2015;4:148-154. https://doi.org/10. 5409/wjcp.v4.i4.148.

6. Husby S, Koletzko S, Korponay-Szabo IR, Mearin ML, Phillips A, Shamir R et al. European Society for Pediatric Gastroenterology, Hepatology, and Nutrition guidelines for the diagnosis of coeliac disease. J Pediatr Gastroenterol Nutr 2012;54:136-160. https:// doi.org/10.1097/MPG.0b013e31821a23d0.

7. Husby S, Koletzko S, Korponay-Szabo I, Kurppa K, Mearin ML, Ribes-Koninckx C et al. European Society Paediatric Gastroenterology, Hepatology and Nutrition Guidelines for Diagnosing Coeliac Disease 2020. J Pediatr Gastroenterol Nutr 2020;70:141-156. https://doi.org/10.1097/MPG.0000000000002497.

8. Murch S, Jenkins H, Auth M, Bremner R, Butt A, France S et al. Joint BSPGHAN and Coeliac UK guidelines for the diagnosis and management of coeliac disease in children. Arch Dis Child 2013;98:806-811. https://doi.org/10.1136/archdischi ld-2013-303996.

9. Riznik P, Balogh M, Bodi P, De Leo L, Dolinsek J, Guthy I et al. The use of biopsy and "no-biopsy" approach for diagnosing paediatric coeliac disease in the Central European Region. Gastroenterol Res Pract 2019;2019:9370397. https://doi.org/10.1155/ $2019 / 9370397$.

10. Penny HA, Sanders DS, Gillett H, Edwards CM. BSG Interim Guidance: COVID-19 specific non-biopsy protocol for those with suspected coeliac disease 2020. Updated 12 Jun 2020. https://www.bsg.org.uk/covid-19-advice/covid-19-specificnon-biopsy-protocol-guidance-for-those-with-suspected-coeli ac-disease/.

11. Hall SW, Day AS. An overview of international guidelines focusing on the long-term management of coeliac disease. Gastrointest Disord 2020;2:152-163. https://doi.org/10.3390/gidis ord2020016.

12. Al-Toma A, Volta U, Auricchio R, Castillejo G, Sanders DS, Cellier C et al. European Society for the Study of Coeliac Disease (ESsCD) guideline for coeliac disease and other gluten-related disorders. United European Gastroenterol J 2019;7:583-613. https://doi.org/10.1177/2050640619844125.

13. Bai JC, Fried M, Corazza GR, Schuppan D, Farthing M, Catassi $\mathrm{C}$ et al. World Gastroenterology Organisation global guidelines on celiac disease. J Clin Gastroenterol 2013;47:121-126. https://doi.org/10.1097/MCG.0b013e31827a6f83.

14. Hill ID, Fasano A, Guandalini S, Hoffenberg E, Levy J, Reilly $\mathrm{N}$ et al. NASPGHAN clinical report on the diagnosis and treatment of gluten-related disorders. J Pediatr Gastroenterol Nutr 2016;63:156-165. https://doi.org/10.1097/MPG.0000000000 001216.

15. Husby S, Murray JA, Katzka DA. AGA clinical practice update on diagnosis and monitoring of celiac disease-changing utility of serology and histologic measures: expert review. Gastroenterology 2019;156:885-889. https://doi.org/10.1053/j.gastro. 2018.12.010.

16. NICE. Coeliac disease: recognition, assessment and management United Kingdom: NICE Guidelines; 2015. Updated January 2020. https://www.nice.org.uk/guidance/ng20/resources/ coeliac-disease-recognition-assessment-and-management-pdf1837325178565.

17. Rubio-Tapia A, Hill ID, Kelly CP, Calderwood AH, Murray JA. ACG clinical guidelines: diagnosis and management of celiac disease. Am J Gastroenterol 2013;108:656-676. https://doi.org/10. 1038/ajg.2013.79.

18. Snyder J, Butzner JD, DeFelice AR, Fasano A, Guandalini $\mathrm{S}$, Liu E et al. Evidence-informed expert recommendations for the management of celiac disease in children. Pediatrics 2016;138:e20153147. https://doi.org/10.1542/peds.2015-3147.

19. Gleeson M, Cripps AW. Development of mucosal immunity in the first year of life and relationship to sudden infant death syndrome. FEMS Immunol Med Microbiol. 2004;42:21-33. https://doi.org/ 10.1016/j.femsim.2004.06.012.

20. Lagerqvist C, Dahlbom I, Hansson T, Jidell E, Juto P, Olcen P et al. Antigliadin immunoglobulin A best in finding celiac disease in children younger than 18 months of age. J Pediatr Gastroenterol Nutr 2008;47:428-435. https://doi.org/10.1097/MPG.0b013 e31817d80f4

21. Khan MR, Silvester JA, Sparks B, Hintze Z, Ediger T, Larson JJ et al. The utility of IgA-based serologic markers in diagnosing celiac disease in children 24 months of age or younger. $J$ Pediatr 2020;224:e2. https://doi.org/10.1016/j.jpeds.2020.04.009.

22. Bishop J, Reed P, Austin P, Hurst M, Ameratunga R, Craigie A et al. Prospective evaluation of the ESPGHAN guidelines for diagnosis of coeliac disease in New Zealand children. J Pediatr Gastroenterol Nutr 2018. https://doi.org/10.1097/MPG.0000000000 002065.

23. Thompson G, Grover Z, Loh R, Mews C, Ravikumara M, Jevon $\mathrm{G}$ et al. Assessment of European Society of Paediatric Gastroenterology Hepatology and Nutrition (ESPGHAN) guidelines in 
an Australian paediatric population. Pathology 2020;52:568-575. https://doi.org/10.1016/j.pathol.2020.05.002.

24. Paul SP, Harries SL, Basude D. Barriers to implementing the revised ESPGHAN guidelines for coeliac disease in children: a cross-sectional survey of coeliac screen reporting in laboratories in England. Arch Dis Child 2017;102:942-946. https://doi.org/10. 1136/archdischild-2016-312027.

25. Ho SSC, Keenan JI, Day AS. Role of serological tests in the diagnosis of coeliac disease in children in New Zealand. $J$ Paediatr Child Health 2020. https://doi.org/10.1111/jpc.15076.

26. Landman M, Theuns SDM, van Wering HM, Tramper G, van Ledden M, Rietveld E et al. Evaluation of the implementation of the 2012 ESPGHAN guideline of Coeliac disease in children: results of a retrospective study in the Netherlands. Arch Dis Child 2020;105:413. https://doi.org/10.1136/archdischild-2018-316490.

27. Werkstetter KJ, Korponay-Szabo IR, Popp A, Villanacci V, Salemme M, Heilig G et al. Accuracy in diagnosis of celiac disease without biopsies in clinical practice. Gastroenterology 2017;153:924-935. https://doi.org/10.1053/j.gastro.2017.06.002.

28. Valitutti F, Trovato CM, Montuori M, Cucchiara S. Pediatric Celiac disease: follow-up in the spotlight. Adv Nutr 2017;8:356361. https://doi.org/10.3945/an.116.013292.

29. Barnea L, Mozer-Glassberg Y, Hojsak I, Hartman C, Shamir R. Pediatric celiac disease patients who are lost to follow-up have a poorly controlled disease. Digestion 2014;90:248-253. https://doi. org/10.1159/000368395.

30. O'Leary C, Wieneke P, Healy M, Cronin C, O'Regan P, Shanahan F. Celiac disease and the transition from childhood to adulthood: a 28-year follow-up. Am J Gastroenterol 2004;99:2437-2441. https://doi.org/10.1111/j.1572-0241.2004.40182.x.

31. Brooks AJ, Smith PJ, Cohen R, Collins P, Douds A, Forbes V et al. UK guideline on transition of adolescent and young persons with chronic digestive diseases from paediatric to adult care. Gut 2017;66:988-1000. https://doi.org/10.1136/gutjnl-2016-313000.

32. Peixoto A, Reis EMA, Trindade E, Dias JA, Macedo G. Protocol for the transition of pediatrics for adult medicine in celiac disease: a proposal approach. Eur J Gastroenterol Hepatol 2019;31:729730. https://doi.org/10.1097/MEG.0000000000001372.

33. Sbravati F, Pagano S, Retetangos C, Spisni E, Bolasco G, Labriola $\mathrm{F}$ et al. Adherence to gluten-free diet in a celiac pediatric population referred to the general pediatrician after remission. J Pediatr Gastroenterol Nutr 2020;71:78-82. https://doi.org/10.1097/MPG. 0000000000002676.

34. Biagi F, Bianchi PI, Marchese A, Trotta L, Vattiato C, Balduzzi $\mathrm{D}$ et al. A score that verifies adherence to a gluten-free diet: a cross-sectional, multicentre validation in real clinical life. $\mathrm{Br} \mathrm{J}$ Nutr 2012;108:1884-1888. https://doi.org/10.1017/S000711451 1007367.

Publisher's Note Springer Nature remains neutral with regard to jurisdictional claims in published maps and institutional affiliations. 